Background Juvenile-onset systemic lupus erythematosus (JSLE) is an autoimmune disorder characterised by immune dysregulation, chronic inflammation and increased cardiovascular risk. Our findings in adult-onset SLE link immune cell dysregulation with defects in plasma membrane signalling platforms (lipid rafts). In JSLE little is known about the immune profile or whether abnormal lipid metabolism contributes to pathogenesis.

Methods Flow cytometry was used to measure metabolic marker expression on immune cell subsets from 39 healthy donors (HCs) and 35 JSLE patients. Metabolic biomarker analysis including lipoprotein composition was performed on matching serum.

Results JSLE patients had significantly elevated membrane lipid rafts in T-cells, B-cells and plasmacytoid dendritic cells compared to HCs suggesting dysregulated membrane receptor signalling. Furthermore, lipid raft expression correlated positively with cell activation markers, disease activity, erythrocyte sedimentation rate and dsDNA titre and negatively with complement protein C3 supporting the hypothesis that altered metabolism is associated with JSLE pathogenesis. Importantly, ROC curve analysis showed that lipid raft expression on these cell types is an excellent diagnostic of high disease activity in JSLE. Metabolomic analysis of matching serum revealed that high disease activity patients had significantly decreased atheroprotective high density lipoproteins (HDL) and increased atherogenic low density lipoproteins (LDL) suggesting altered transport of lipids. In addition, lipids associated with membrane rafts such as sphingomyelin, phosphatidylcholine, phosphoglycerides and cholesterol correlated negatively with HDL in high disease activity patients but positively in low disease activity patients. Immune cell lipid rafts correlated positively with LDL and negatively with HDL together suggesting altered lipid uptake/efflux from these cells; this may alter immune cell signalling in JSLE patients. Stratification of patients based on their lipid profile by hierarchical clustering revealed 3 groups that were unique in both immnophenotype and clinical presentation.

Conclusions Differences in the metabolic profiles of immune cell subsets and lipoprotein lipid transport in JSLE contribute to disease pathogenesis and severity. Regulation of lipid metabolism may therefore have therapeutic benefit for JSLE patients providing a dual effect of reducing inflammation and atherosclerotic risk. These therapeutics may perform better in patients that present specific clinical and phenotypic features.

\section{PS5:88 ALTERED CELL SURFACE N-GLYCOSYLATION OF AUTOIMMUNE T-CELLS RESULTS IN DECREASED BINDING OF IMMUNOREGULATORY GALECTIN-1}

'L Kovács, ${ }^{2} E$ Szabó, ${ }^{1,2} A$ H Hornung, ${ }^{2} E ́$ Monostori, ${ }^{2} A$ Czibula. ${ }^{1}$ University of Szeged, Faculty of Medicine, Dept of Rheumatology and Immunology, Szeged, Hungary; ${ }^{2}$ Biological Research Centre of the Hungarian Academy of Sciences, Dept of Genetics, Lymphocyte Signal Transduction Lab, Szeged, Hungary

10.1136/lupus-2018-abstract.133

Glycosylation (sugar code) is frequently altered on the surface of various cells in patients with immune-mediated diseases resulting in changes in signal transduction and metabolic control. However, alteration of glycosylation patterns of $\mathrm{T}$ cells has not yet been characterised in autoimmune disorders. We have previously demonstrated that T-cells of patients with systemic lupus erythematosus (SLE) are resistant to the apoptotic effects of galectin-1 (Gal-1), an immunoregulatory lectin.

T-cells from patients with active SLE $(n=18)$, rheumatoid arthritis (RA) $(n=14)$ or Sjögren's syndrome (SS) $(n=14)$ and from healthy controls $(n=19)$ were examined. Cell surface glycosylation was analysed with lectin-binding assay, and the expression of glycosyltransferase and glycosidase enzymes participating in the construction of the glycan chains was measured with reverse transcriptase polymerase chain reaction (RTPCR).

Resting SLE T-cells bound significantly higher amounts of several lectins, while RA and SS resting T-cells were similar to controls in their glycosylation pattern. Activated T-cells from all autoimmune groups bound significantly less Gal-1 than controls, while other lectins bound similarly. Gene expression including alpha mannosidases (MAN1A1, MAN1A2, MAN2A1 and MAN2A2) and beta-N-acetylglucosaminyltransferases (MGAT1-5) in autoimmune activated T-cells did not differ from controls with the only exception of MAN1A2 in SS. However, we found a significant increase in the mRNA ratios of certain sialyltransferases and neuraminidases, specifically in ST6GAL1/NEU1 in SLE and SS, and in ST3GAL6/NEU1 in SLE and RA patients compared to controls. Treatment of cells with neuraminidase resulted in a remarkable increase in Gal-1 binding

The glycosylation pattern of resting SLE T-cells was consistent with a pre-activated phenotype. Decreased Gal-1 binding found in all three diseases can be explained with an increased terminal sialylation, which may be a consequence of an altered expression of sialyltransferase and neuraminidase genes, whose concerted action is responsible for the degree of sialylation of glycan structures. Accordingly, neuraminidase treatment resulted in remarkably increased Gal-1 binding. We propose that increased sialylation may at least partially explain the previously found resistance to the immunoregulatory effects of Gal-1 in SLE, hence contributing to the pathomechanism of the examined diseases.

\section{PS5:89 LOOKING FOR A SLE SIGNATURE ON PERIPHERAL B CELL SUBSETS: DOES A PREPONDERANT CD38 POSITIVE PLASMABLAST-SUBPOPULATION LACK CD73 AS A SIGN OF A DIMINISHED B REGULATORY POOL?}

M Siekierka-Harreis, M Schroedter, G Chehab, J Richter, S Vordenbäumen, M Schneider, G Pongratz. Rheumatology, Medical Faculty Heinrich-Heine University, Düsseldorf, Germany

\subsection{6/lupus-2018-abstract.134}

Background Systemic Lupus Erythematosus (SLE) is an autoimmune disorder characterised by polyclonal Bcell activation, production of dsDNA-autoantibodies and cytokines. Subsets of Bcells play a central role in SLE-pathogenesis. The inflammatory milieu is characterised by the accumulation of adenosine, which confers immunosuppressive effects. In SLE, the role of CD73, an enzyme involved in the extracellular generation of adenosine from ATP, is not well characterised. This study aimed to characterise expression of CD73 B cell subsets of SLE-patients as compared to healthy controls (HC).

Methods B cell subsets were characterised from peripheral blood of 23 SLE patients attending the outpatient clinic at the Rheumatology Unit of University Hospital Düsseldorf and of $15 \mathrm{HC}$ by FACS. All patients fulfilled the revised SLE-criteria 